\title{
Research on SIFT Algorithm Base on Complex Environment
}

\author{
Li dan ${ }^{1,2, a}$, Dang xiangying ${ }^{1,2}$ \\ ${ }^{1}$ Xuzhou Institute of Technology, Xuzhou, China \\ ${ }^{2}$ Xuzhou Machine Vision Application Engineering Technology Research Center, Xuzhou, China \\ aE-mail: lidanonline@163.com
}

Keywords: traffic; stitching; Curvelet; SIFT

\begin{abstract}
A new algorithm of feature matching was presented by this paper. In the new algorithm, Curvelet first used to denoise, then RANSAC optimized SIFT algorithm used to stitch images. Experiments show that the new algorithm is better to remove error matching points. It improved the accuracy of local invariant feature matching. New algorithm has a good anti-noise and lighting capabilities. It has strong adaptability to scale changes, the algorithm is robust, and has better effect on matching and stitching.
\end{abstract}

\section{Introduction}

Firstly, the paper used Curvelet algorithm to remove image noise, then used SIFT scale invariant feature vectors to match features. The improved Ransac method was used to remove the mismatch. The new algorithm is adapted to the complex environment with noise, light changes, blur and rotation problems.It is robust and accuracy while ensuring the matching efficiency.

\section{Image Preprocessing}

Due to the problems of complex environmental monitoring video has noise, low resolution. In order to accurately extract the feature points, need image preprocessing to remove noise. Wavelet has good time-domain and frequency-domain analysis capabilities, many scholars have proposed a method based on wavelet denoising, such as nonlinear thresholding wavelet transform, translation invariant denoising, etc. However, the two-dimensional separable wavelet has only a limited level, and diagonal direction, because of lack of multi-direction make it can not represent a line of exotic, bizarre face high dimensional function. Based on wavelet theory, E.J.Candes and D.L.Donoho established a ridge wave transformation from 1998 to 1999 [1]. Ridge wave uses a wavelet function by adding characterized direction parameters. It has a strong direction selection and the ability to identify and can effectively represent the direction singular characteristic in signal. To further indicate the multidimensional signal general curved singularities, and the Curvelet methods [2] can use the multi-scale local linear approximation of the curve. Noise and edge information can be well separated, while keeping an edge, the noise suppression achieved good results. This paper will apply the second generation Curvelct transform in image denoising, it will have higher image SNR and the image will display more clearly.

A. Discrete Curvelet

$f\left[t_{1}, t_{2}\right], 0 \leq t_{1}, t_{2} \leq n$ is the input function,Curvelet transformation [3] can be expressed as: $\quad c^{D}(j, l, k):=\sum_{0 \leq t_{1}, t_{2}<n} f\left[t_{1}, t_{2}\right] \overline{\varphi_{j, l, k}^{D}\left[t_{1}, t_{2}\right]}$. Using bandpass function $\Psi\left(\omega_{1}\right)=\sqrt{\phi\left(\omega_{1} / 2\right)^{2}-\phi\left(\omega_{1}\right)^{2}}$, definite $\Psi_{j}\left(\omega_{1}\right)=\Psi\left(2^{-j} \omega_{1}\right)$ to achieve multi-scale segmentation. For each $\omega=\left(\omega_{1}, \omega_{2}\right), \quad \omega_{1}>0$, it has $V_{j}\left(S_{\theta_{1}}(\omega)=V\left(2^{[j / 2} \frac{\omega_{2}}{\omega_{1}}-l\right) \cdot S_{\theta l}\right.$ is a shear matrix $S_{\theta l}:=\left(\begin{array}{cc}1 & 0 \\ -\tan \theta_{l} & 1\end{array}\right)$. Definite $\theta_{j}(\omega):=\psi_{j}\left(\omega_{1}\right) V_{j}(\omega)$, for each $\theta_{1} \in[-\pi / 4, \pi / 4)$, it has $\dot{\theta}_{j, 1}(\omega):=\psi_{j}\left(\omega_{1}\right) V_{j}\left(S_{\theta_{1}}(\omega)=U_{j, 1}\left(S_{\theta_{1}}()\right)\right.$.

Discrete Curvelet transform first transformed to the frequency domain, then localized in the frequency domain, after localization, using 2DIFFT to get Curvelet coefficients.

$B$. Curvelet Based on the USFFT 
(1) For a given two-dimensional function $f\left[t_{1}, t_{2}\right], 0 \leq t_{1}, t_{2} \leq \omega$ get on 2DFFT [4,5],obtain a two-dimensional frequency domain representation: $f\left[n_{1}, n_{2}\right],-n / 2 \leq n_{1}, n_{2} \leq n / 2$;

(2) In the frequency domain, for each pair (j,1\}), resampling $f\left[n_{1}, n_{2}\right]$, obtain samples $f\left[n_{1}, n_{2}-n_{1} \tan \theta_{1}\right] \quad, \quad\left(n_{1}, n_{2}\right) \in P_{j}$, among them $P_{j}=\left\{\left(n_{1}, n_{2}\right): n_{1,0} \leq n_{1}<n_{1,0}+L_{1, j}, \quad n_{2,0} \leq n_{2}<n_{2,0}+L_{2, j}\right\}$ and $L_{1, j}$ is about $2^{j}, L_{2, j}$ is the parameters on the $2^{j / 2}$. Denote the support interval length, width of window function $\theta_{j}\left[n_{1}, n_{2}\right]$;

(3) The interpolated $f$ multiply the window function $\theta_{\mathrm{j}}$ get $f_{j, 1}\left[n_{1}, n_{2}\right]=f\left[n_{1}, n_{2}-n_{1} \tan \theta_{1}\right] \Theta_{j}\left[n_{1}, n_{2}\right]$;

(4) 2DIFFT inverses transformation performed on $\uparrow_{j, 1}$ and obtained discrete Curvelet coefficient $C^{D}(j, l, k) \cdot$

\section{Feature Detection}

SUSAN, Harris corner detection operators with certain degree of invariance, but there are more sensitive to noise and scale changes, so not suitable for viewing angle changes, low-light and high-noise environments. Local scale-invariant SIFT algorithm uses a multi-scale transform in scale space to find extreme points. Extracting feature points position, so that the direction of feature vectors remains unchanged.

A. SIFT Feature point detection and location

In the multi-scale to detect space feature points, image scale space definited as the following: $L(x, y, \sigma)=G(x, y, \sigma) * I(x, y)$ 。

SIFT operator uses Gaussian differential operator to look for great value in the scale space to detect feature points, as shown in Figure 1, differential equations for Gaussian scale space is:

$D(x, y, \sigma)=(G(x, y, k \sigma)-G(x, y, \sigma)))^{*} I(x, y)=L(x, y, k \sigma)-L(x, y, \sigma)$

$$
G(x, y, \sigma)=\frac{1}{\sqrt{2 \pi} \sigma} e^{-\left(x^{2}+y^{2}\right) / 2 \sigma^{2}}
$$

The middle detection points compares with 8 neighbor points of the same scale, and the adjacent 18 points , ensure extreme points in the two-dimensional image and scale space can be detected. As shown in Figure 2:

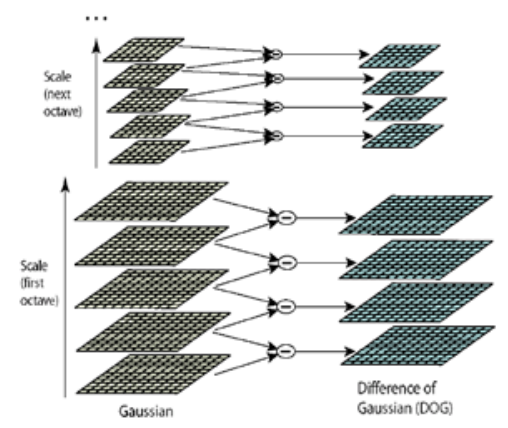

Figure1. Difference of Gaussian space

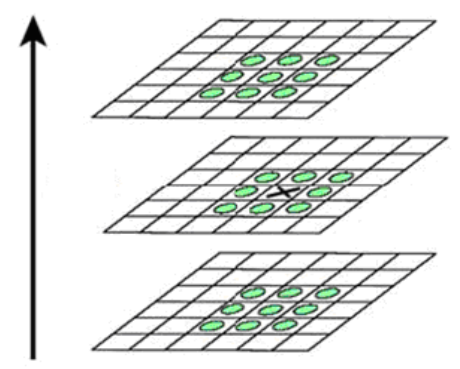

Figure2. Threshold detector

B. Distribution of the main directions of the key point

Each key specified the direction parameter by neighborhood pixels gradient direction distribution, 
ensure that operators have a rotation invariant .

$$
\begin{aligned}
& m(x, y)=\sqrt{(L(x+1, y)-L(x-1, y))^{2}-(L(x, y+1)-L(x, y-1))^{2}} \\
& \theta(x, y)=\alpha \tan 2((L(x, y+1)-L(x, y-1)) / L(x+1, y)-L(x-1, y))
\end{aligned}
$$

Where: $m(x, y), \theta(x, y)$ represent the modulus value and direction of sampling point, statistics gradient direction by histogram. Peak represents the main direction of the key points.

C. Feature vector generation

Axis rotates to the direction of the feature region, the key point as the center use $16 \times 16$ window, each $4 \times 4$ calculates 8 gradient directions histogram on small pieces. Draw the accumulated value of each gradient direction. One of the key point constituted from the seed point of 16 seed points. Each feature point uses a 128-dimensional vector characterization.

\section{Optimized Match}

After comparing the feature points found by SIFT algorithm with all the feature points to be matched, calculating the euclidean distance and determining similarity measure. If the distance between the nearest and next nearest is less than the threshold value, such as 0.5 , then start matching. But under the harsh environment, there will be a mismatch phenomenon. It needs to filter out the right feature matching points for post-splicing.

RANSAC (Random Sample Consensus) is an iterative algorithm method to estimate model parameters from a set of observed data, the algorithm has a random uncertainty. It can increase the probability of correct model solved by increasing the number of iterations. The results obtained may be different each time, but always gives a reasonable result. Only four pairs of feature points will find the transformation matrix model. RANSAC specific algorithm is as the following:

1)Randomly selecte four pairs of matching features for computing the transformation matrix;

2)Feature pairs are divided into inner and outer points according to the transformation matrix;

3)If the optimal solution of the characteristics which meet the requirements are more than the current matrix, then update the transformation matrix;

4)Repeat step 1) until the specified number of iterations .

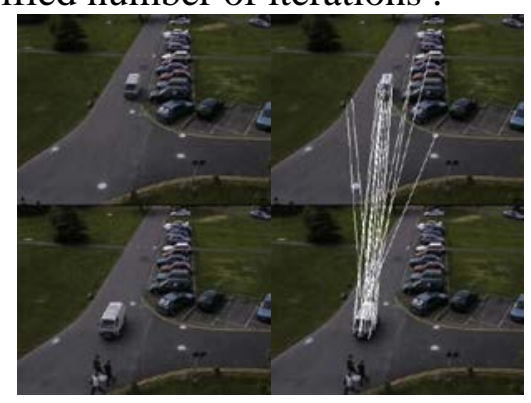

(a)

(b)

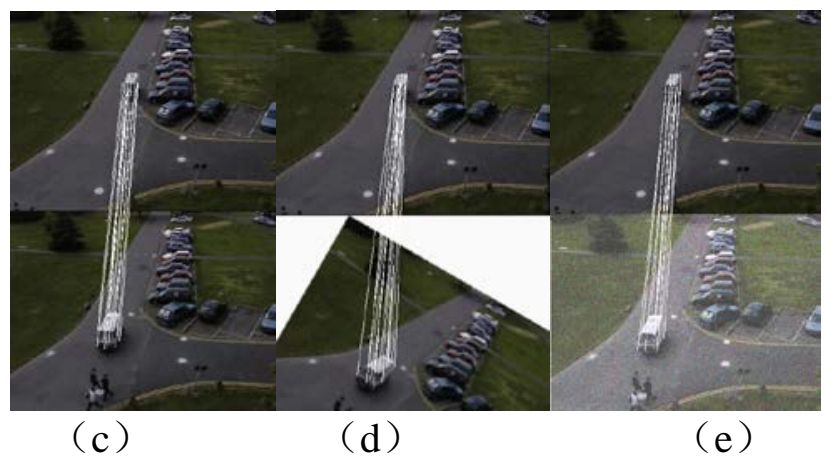

Figure 3. Matching results in different environments

Figure3 (a) is a picture to be matched, (b) is the result of the original SIFT matching algorithm, as can be seen, there are a lot of mismatching. Figure (c) is optimized through this article, after getting rid of mismatching points the feature matching results, mismatching substantial reduced. Figure (d) is the second picture after obfuscation rotation angle match. Figure (e) is the matching results after 
the second picture obfuscation and rotate a certain angle. Good results were obtained. Table 1 describes the change under different environments correct matching rate. Thus, this paper improved SIFT local invariant feature extraction algorithm matches accuracy in a complex environment is improved and has a good prospects.

TABLE 1 MATCH RATE（\%)

\begin{tabular}{cccccc}
\hline & blur & $\begin{array}{c}\text { Brightness } \\
\text { enhancement }\end{array}$ & rotation & noise & scaling \\
\hline SIFT & 72 & 68 & 64 & 62 & 73 \\
\hline This paper & 93 & 90 & 83 & 87 & 96 \\
\hline
\end{tabular}

\section{Splice}

Image mosaic is one application direction of SIFT feature matching. By extracting matching feature points, it can create the following projection transformation model, the dispersion of different perspectives, different sizes, different illumination images spliced into the whole image. Expanding the field of view and can better to capture video information. Eight parameters projection transformation model established according to the two images match points:

$$
\left[\begin{array}{c}
x_{a} \\
y_{a} \\
1
\end{array}\right]=H\left[\begin{array}{c}
x_{b} \\
y_{b} \\
1
\end{array}\right]=\left[\begin{array}{lll}
h_{1} & h_{2} & h_{3} \\
h_{4} & h_{5} & h_{6} \\
h_{7} & h_{8} & 1
\end{array}\right]\left[\begin{array}{c}
x_{b} \\
y_{b} \\
1
\end{array}\right]
$$

In the process of splicing of two images, assume that $a$ and $b$ are the corresponding two points of image $\mathrm{A}$ and the matching image $\mathrm{B}$. $\mathrm{H}$ is the projective transformation matrix. Homography transformation matrix $\mathrm{H}$ can be described as a series of zoom, rotate, pan operation and perspective effects. $\mathrm{h}$ is the parameter vector of $\mathrm{H}$. If getting four right pairs of matching points, you can solve the projection transformation parameters. Splicing effect is as follows:

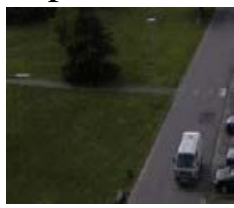

(a)

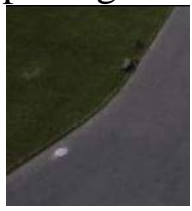

(b)

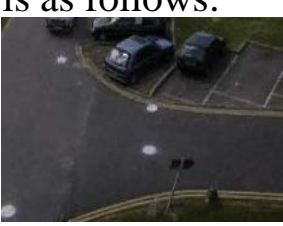

(c)

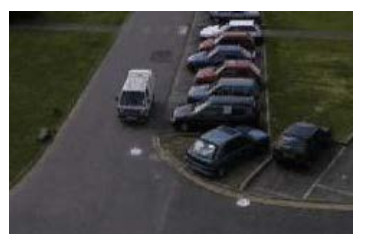

(d)

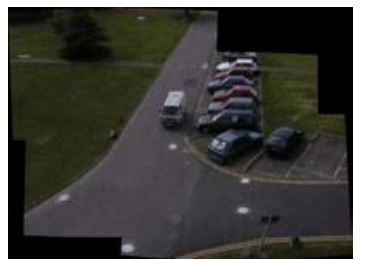

( e )

Figure4. Splicing

Figure 4 (a)-(d) are three images of a scene, Figure 4 (e) is the result after stitching. It can be seen that the algorithm by removing the mismatch feature points to splic. In low illumination, high noise environments mosaic effect is also perfect.

\section{Conclusion}

Due to the complexity of the traffic environment, the commonly used corner detection operator is sensitive to noise, light and changes in scale conditions. New features matching algorithm proposed after de-noising by Curvelet, the SIFT algorithm using RANSAC to stitch. Experiments showed that the new algorithm can better removing the error matching points, improve the accuracy of local invariant feature matching. It has a good anti-noise and lighting capabilities and adaptability to the changes in scale. The new algorithm is robust and the matching, stitching results are better. It has robustness in a complex environment with high noise and has a good prospect. 


\section{Acknowledgment}

This work is partly supported by the Ministry of Housing and Urban Construction Science and Technology Plan Project(2014-K5-027) and Xuzhou Machine Vision Application Engineering Technology Research Center.

\section{References}

[1]Candes E J. Ridgelet:Theory and Applications [D].USA : Department of Statistics,Stanford University,1998.

[2]E.J.Candes,D.L.Donoho.Curvelets[D].USA :Department of Statistics, Stanford University,1999

[3]Candes, Emmanuel; Demanet, Laurent; Donoho, David; Ying, Lexing. Fast discrete curvelet transforms [J] .Multiscale Modeling and Simulation, 2006 ,5(3):861-8991

[4]CAND’ES E J,DEMANET L.Curvelets and fast wave equation solvers[D].USA:California Institute of Technology,2005.

[5]E J Candes,D L Donoho.New tight frames of curvelets and optimal representations of objects with C2 singularities[J].Commun. on Pure and Appl.Math. 2004,57(2):219-266 